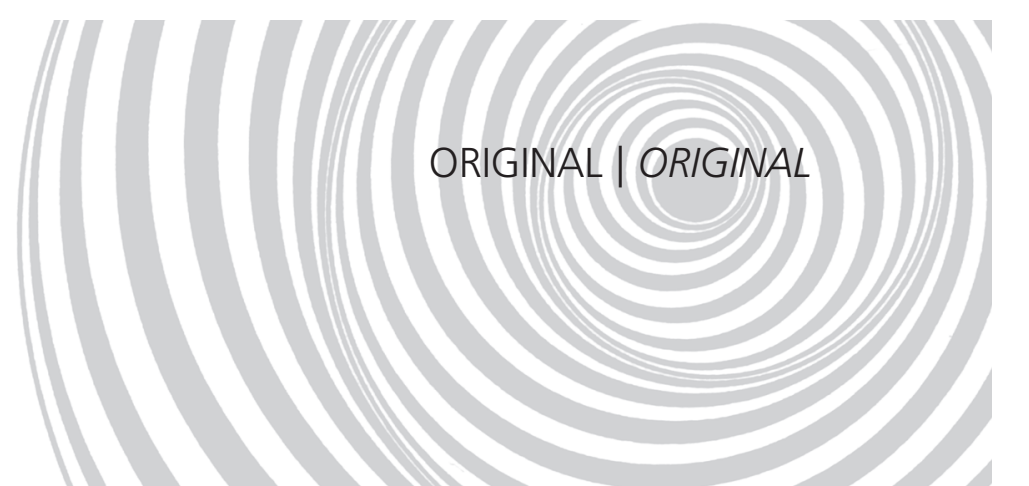

\title{
Descrição sócio-demográfica, laboral e de saúde dos trabalhadores do setor de serviços de alimentação dos restaurantes populares do estado do Rio de Janeiro ${ }^{1}$
}

\author{
Sociodemographic, labor, and health characteristics \\ of workers from popular foodservices in the
}

state of Rio de Janeiro, Brazil

Odaleia Barbosa de AGUIAR ${ }^{2}$

Joaquim Gonçalves VALENTE ${ }^{3}$

Maria de Jesus Mendes da FONSECA ${ }^{3}$

RE S U M O

\section{Objetivo}

Este artigo tem o objetivo de descrever uma população de trabalhadores de alimentação coletiva com foco nos perfis sócio-demográfico, laboral e de saúde.

\section{Métodos}

O estudo, do tipo seccional, foi realizado com 426 trabalhadores dos restaurantes populares do Estado do Rio de Janeiro, com um questionário aplicado por entrevistadores treinados. Os testes para fins de verificação de associação foram o qui-quadrado de Pearson, e, alternativamente, o teste exato de Fisher para amostras pequenas.

\section{Resultados}

Os homens representaram $62,7 \%$ do total de trabalhadores. A idade média dos funcionários foi de 35,1 anos, $(\mathrm{DP}=10,3)$. Quanto à escolaridade, somente $11,3 \%$ possuíam escolaridade menor que quatro anos e $42,2 \%$ entre cinco e oito anos de escolaridade. Os incômodos ambientais apresentaram a seguinte ordem decrescente de importância na percepção dos trabalhadores: temperatura $(90,1 \%)$, ruído $(51,2 \%)$, esforço físico $(36,2 \%)$ e luminosidade (10,5\%). Os relatos de doenças com diagnóstico médico apresentaram prevalência de: 15,0\%

1 Artigo elaborado a partir da tese de O.B. AGUIAR, intitulada "Aspectos psicossociais do impedimento laboral por motivos de saúde em trabalhadores de cozinhas industriais". Universidade do Estado do Rio de Janeiro; 2009.

2 Universidade do Estado do Rio de Janeiro, Instituto de Nutrição, Departamento de Nutrição Aplicada. R. São Francisco Xavier, 524, Sala 12026, Bloco D, Maracanã, 20550-013, Rio de Janeiro, RJ, Brasil. Correspondência para/Correspondence to: O.B. AGUIAR. E-mails: <odaleia@uerj.br>; <odaleiab@hotmail.br>.

${ }^{3}$ Fundação Oswaldo Cruz, Escola Nacional de Saúde Pública, Departamento de Epidemiologia. Rio de Janeiro, RJ, Brasil. 
970 | O.B. AGUIAR et al.

\begin{abstract}
"doença osteomusculares relacionadas ao trabalho (DORT)"; 14,3\% hipertensão arterial sistêmica; 12,7\%, gastrite e 2,1\%, diabete Mellitus tipo II. Em relação aos acidentes de trabalho 20,2\% relataram ter sofrido corte, seguido de contusão com 16,0\%, nos últimos doze meses.
\end{abstract}

\title{
Conclusão
}

A análise dos dados permitiu concluir que, embora os diferentes cargos sofram a ação do ambiente de trabalho, da remuneração e da qualificação, estas questões estiveram mais associadas aos auxiliares de serviços gerais.

Termos de indexação: Alimentação coletiva. Ambiente de trabalho. Condições de trabalho. Trabalhadores.

\section{A B S T R A C T}

\section{Objective}

This study described a population of foodservice workers, focusing on their sociodemographic, labor and health characteristics.

\section{Methods}

This sectional study included 426 (98\%) workers of popular foodservices of the state of Rio de Janeiro who answered a questionnaire administered by trained interviewers. Associations were investigated with the Pearson's chi-square test or the Fisher's exact test for small samples.

\section{Results}

Most of the workers were males (62.7\%). The mean age of the workers was 35.1 years (SD=10.3). Very few workers (11.3\%) had had less than 4 years of formal education and $42.2 \%$ had had from 5 to 8 years of formal education. The following environmental stressors were mentioned with decreasing order of importance: temperature (90.1\%), noise (51.2\%), physical effort (36.2\%) and luminosity (10.5\%). The medical diagnoses included work-related musculoskeletal disorders (15.0\%), hypertension (14.3\%), gastritis (12.7\%) and diabetes mellitus type II (2.1\%). The most common work accidents in the 12 months prior to the interview were cuts (20.2\%) and bruises (16.0\%).

\section{Conclusion}

The data showed that although different jobs are affected by the work environment, wages and qualification, these factors had a greater impact on the general service assistants.

Indexing terms: Collective feeding. Working environment. Working conditions. Workers.

\section{N T R O D U Ç Ã O}

Desde os anos oitenta e mais intensamente na década de noventa, as mudanças estruturais no mercado de trabalho em economias mundiais tiveram como reflexo uma maior concentração de trabalhadores no setor de serviços. O aumento relativo de pessoal ocupado no setor terciário verificou-se tanto em ocupações técnicas, administrativas e gerenciais, quanto naquelas com menor exigência de conhecimentos tecnológicos, com baixa qualificação e com menor remuneração. Dentre esses segmentos, encontram-se os serviços: comunitários, de reparação, de hospedagem e alimentação, de limpeza e serviços domésticos ${ }^{1-3}$.
A expansão do segmento de alimentação tem se beneficiado com o aumento do número de pessoas que se alimentam fora de casa. Em estudos realizados nos Estados Unidos da América, os autores apontaram para uma tendência no aumento do número de refeições realizadas fora de casa ${ }^{4-6}$.

Os resultados da Pesquisa de Orçamentos Familiares (POF), realizada em 2002 e 2003 pelo Instituto Brasileiro Geografia e Estatística (IBGE) revelaram mudanças no comportamento das famílias brasileiras em relação ao local de realização das principais refeições, pois, aproximadamente $24 \%$ da despesa média mensal familiar com alimentação foram destinados a refeições fora de casa?. 
No ano de 2008, a Associação Brasileira das Empresas de Refeições Coletivas (ABERC) contabilizou 180 mil empregados diretamente no setor, um fornecimento de 13,7 milhões de refeições/dia pelas empresas prestadoras de serviços e um faturamento de 9,5 bilhões de reais no ano ${ }^{8}$.

$O$ atendimento deste aumento de demanda de refeições deve considerar hábitos alimentares dos consumidores, disponibilidade de fornecedores de alimentos, ambiente de trabalho e mão-de-obra para produzir grandes quantidades de refeições. Uma unidade de alimentação e nutrição produz refeições nutricionalmente equilibradas, podendo reunir um grande número de trabaIhadores, em função do número de refeições servidas, e da diversidade de produtos e serviços oferecidos $^{9}$.

A absorção de trabalhadores por esse recente mercado de trabalho não encontra entraves na idade (não é comum o estabelecimento de idade mínima ou máxima para contratação de pessoal), sexo, ter concluído o ciclo de educação fundamental, possuir qualificação específica na área ou demonstração de qualquer habilidade estabelecida a priori. É senso comum que esse setor apresenta problemas de gestão de recursos humanos. Geralmente, as dificuldades dos trabalhadores na aprendizagem de rotinas relacionadas à manipulação de alimentos, padronização dos processos de higiene e interesse no seu desenvolvimento são atribuídas a baixas ou deficientes escolaridade e certificação de qualificação profissional10,11.

O setor de alimentação coletiva é um setor percebido como "de passagem", em que os indivíduos ficam pouco tempo; apresenta alta rotatividade, alto absenteísmo e baixa atratividade, causados pelas limitações quanto à motivação e ao reconhecimento profissional ${ }^{10,11}$. O baixo status profissional dos trabalhadores impulsiona o declínio da remuneração e, nesta mesma direção, os salários pressionam pela diminuição da qualidade da mão-de-obra do setor.

Adicionalmente à insuficiente qualificação dos trabalhadores de alimentação coletiva, o pro- cesso de trabalho é executado rotineiramente sob condições ambientais de ruído excessivo, temperatura elevada, iluminação precária, ventilação insatisfatória, além de serem utilizados equipamentos quebrados ou em condições arriscadas de funcionamento, o que pode estar diretamente relacionado tanto à produtividade como à saúde dos trabalhadores ${ }^{9,10,12-14}$.

Associadas às questões ambientais, as atividades de preparo de alimentos, na maioria dos casos, são desenvolvidas, em intenso ritmo e esforço, postura de pé na maior parte do tempo de trabalho e movimentos repetitivos e monótonos. Além disso, exige-se polivalência dos operadores, alocando-os a um número elevado de tarefas diferentes, de acordo com as necessidades do momento e a pressão gerada pelos horários em que as refeições devem ser servidas ${ }^{9,12-14}$.

Se pouca atenção tem sido dada ao ambiente do setor de alimentação coletiva e aos procedimentos das tarefas com o intuito de promover o bem estar dos trabalhadores, menos atenção ainda tem sido direcionada aos agravos à saúde decorrentes do processo de trabalho em produção de refeições. A situação de saúde dos trabalhadores de restaurantes industriais aponta uma alta frequência de Doenças Osteomuscular Relacionadas ao Trabalho (DORT), como a mais frequentemente encontrada, seguida de dor na coluna, doenças venosas, doenças do aparelho digestivo, distúrbios psiquiátricos, doenças reumáticas e alergias ${ }^{15-17}$.

Atualmente, pequenos grupos de pesquisadores ligados a área de alimentação coletiva vem desenvolvendo estudos $s^{9,10,14}$ com objetivo de conhecer aspectos desse setor de serviços que tem contribuído para a geração de postos de trabalho no Brasil, com expansão média anual de 1,5\% nos últimos cinco anos ${ }^{8}$. Nessa mesma linha, o objetivo deste estudo foi descrever uma população de trabalhadores de alimentação coletiva com foco nos perfis sócio-demográfico, laboral e de saúde, visando analisar o potencial da mão-de-obra operacional e contribuir para a busca de soluções para problemas gerenciais. 


\section{MÉ TOD OS}

Trata-se de um estudo seccional realizado nos nove Restaurantes Populares, localizados no Estado do Rio de Janeiro e administrados pelo Governo do Estado, em funcionamento no ano de 2006: três no município do Rio de Janeiro, nos bairros de Bangu, Central do Brasil e Maracanã, e um em cada um dos municípios de: Barra Mansa, Campos dos Goytacazes, Duque de Caxias, Itaboraí, Niterói e Nova Iguaçu.

A pesquisa teve como sujeitos todos os trabalhadores que faziam parte do quadro profissional das empresas que detinham a concessão para a produção de refeições nos restaurantes populares; em apenas dois restaurantes, as empresas responsáveis eram as mesmas.

Em dezembro de 2006, os nove restaurantes populares totalizavam 435 trabalhadores, recenseados segundo as categorias profissionais consideradas para o presente estudo: nutricionista, auxiliar administrativo, caixa, estoquista, auxiliar de estoque, chefe de cozinha, cozinheiro, ajudante de cozinha, magarefe, auxiliar de magarefe, copeiro e auxiliar de serviços gerais. Os critérios de exclusão foram: os trabalhadores contratados como orientadores de fila, vigias e seguranças patrimoniais, por não participarem diretamente do processo produtivo de refeições e, em consequência, não estarem expostos às condições ocupacionais comuns às demais categorias. Além desses, os nutricionistas e administradores que representavam o Estado, e aqueles que estavam licenciados por motivos de saúde ou licença maternidade também foram excluídos.

Foram realizadas 426 entrevistas, o que representa uma perda de, apenas, nove trabalhadores $(2,0 \%)$.

As variáveis utilizadas na descrição do perfil dos trabalhadores de alimentação coletiva foram:

- Sócio-demográficas: sexo, idade, autoclassificação de cor de pele, anos de escolaridade, renda mensal familiar líquida em salários-mínimos, situação conjugal, situação residencial, filhos, hábito de fumar, uso de bebida alcoólica.
- Ocupacionais: cargo atual ocupado, tempo de trabalho em cozinhas, se usufrui de horário de almoço, postura corporal para realização das tarefas, cursos realizados na área de alimentação coletiva, treinamento recebido no local de trabalho para ocupar o cargo, percepção relatada pelo trabalhador sobre os incômodos ambientais (temperatura, ruído, esforço, luminosidade) e relacionados à chefia imediata.

- Situação de saúde: estado geral de saúde em comparação a pessoas de sua idade, relato de presença de doenças diagnosticadas por médico (hipertensão arterial, diabetes Mellitus, doenças osteomusculares relacionadas ao trabalho - DORT e gastrite), acidentes de trabalho ocorridos nos últimos 12 meses (corte, queimadura, choque elétrico, perfuração por objeto e contusão), e a classificação de presença ou ausência de transtornos mentais comuns por meio de respostas à versão abreviada do General Health Questionnaire ${ }^{18}$.

A coleta de dados foi realizada no período de dezembro de 2006 a março de 2007, no próprio local de trabalho, onde foi disponibilizada uma sala que proporcionava conforto e privacidade ao indivíduo durante a entrevista. A sensibilização dos sujeitos da pesquisa foi realizada através de informativo, distribuído diretamente a eles, e cartazes afixados no setor de trabalho.

Os questionários foram aplicados por seis alunos do Curso de Graduação em Nutrição da Universidade do Estado do Rio de Janeiro. Estes foram previamente treinados e submetidos à dinâmica, com o objetivo de avaliar a comunicação e compreensão da pesquisa. Foram simuladas situações passíveis de ocorrerem no desenrolar do trabalho, e o manual do entrevistador foi lido em voz alta, em passos lentos e todas as dúvidas foram amplamente discutidas. Durante todo o processo enfatizava-se a importância da participação dos trabalhadores na entrevista, objetivando obter respostas mais próximas possíveis da realidade vivenciada por eles, e atenuar as recusas. As visitas eram agendadas com os nutricionistas dos restaurantes. 
Os dados foram digitados em uma planilha (Microsoft ${ }^{\circledR}$ Excel-2003). As variáveis consideradas neste artigo foram: 1) idade: anos completos até 29 anos, de 30 a 44 anos e mais de 45 anos; 2) cor: branca, parda e negra; 3) escolaridade: menor ou igual a quatro anos, entre cinco e oito anos, e maior ou igual a nove anos; 4) renda: até um salário-mínimo, maior que um e menor ou igual a dois salários-mínimos, e maior do que dois salários-mínimos; 5) situação conjugal: solteiro, casado e separado/viúvo; 6) situação residencial: alugada ou própria; 7) hábito de fumar: não fumante, ex-fumante e fumante; 8 ) tempo de trabalho em cozinhas, em meses; 9) postura corporal: em pé ou sentado; 10) estado geral de saúde: muito bom, bom, regular e ruim; 11) transtornos mentais comuns: presença de três ou mais positividades e as dicotômicas; 12) uso de bebida alcoólica; 13) números de filhos; 14) horário de almoço; 15) cursos; 16) treinamento; 17) incômodos ambientais (temperatura, ruído, esforço, luminosidade); 18) incômodo com chefia; 19) hipertensão arterial; 20) diabetes Mellitus; 21) DORT; 22) gastrite; 23) corte; 24) queimadura; 25) choque elétrico; 26) perfuração por objeto; 27) contusão.

As categorias ocupacionais foram construídas agrupando-se os cargos e considerando-se a semelhança das tarefas no processo de trabalho, como por exemplo, atividades de manipulação dos alimentos, envolvendo os cargos de: copeiros, auxiliares de cozinha e cozinheiros; ou aqueles que não estão diretamente relacionados ao preparo dos alimentos como os nutricionistas, caixas, estoquistas e auxiliares administrativos, resultando, assim, em três grupos: (a) auxiliares de serviços gerais (ASG), (b) copeiros + auxiliares de cozinha + cozinheiros (COP/COZ) e (c) nutricionistas, caixas, estoquistas e Auxiliares Administrativos (ADM). Embora os nutricionistas fossem uma categoria diferenciada dos demais, a opção de agrupá-las aos administrativos deveu-se, principalmente, ao pequeno número na população estudada.

$\mathrm{Na}$ análise dos dados foi utilizado o software Stata versão 9.0.19. Foram realizadas análises univariadas, bivariadas e estratificadas para fins de verificação de associações. Foi aplicado o teste qui-quadrado de Pearson, e alternativamente o teste exato de Fisher para amostras pequenas, para verificar se as associações encontradas apresentavam o nível de significância prefixado em 0,05.

A pesquisa foi submetida, previamente, ao Comitê de Ética em Pesquisa do Instituto de Medicina Social da Universidade do Estado do Rio de Janeiro (CEP-IMS) protocolo 15/5006 em 22 de novembro de 2006, e todos os participantes assinaram o Termo de Consentimento Livre e Esclarecido antes de sua inclusão na amostra.

\section{RE S U L T A DOS}

Os Restaurantes Populares situados em Bangu, Central do Brasil, Duque de Caxias, Maracanã, Niterói e Nova Iguaçu possuíam em média 57 trabalhadores e distribuíam 3 mil refeições/dia; os de Barra Mansa, Campos dos Goytacazes e Itaboraí, 33 trabalhadores e 1500 refeições/dia.

As caracterizações sócio-demográfica e laboral da população estão descritas na Tabela 1 . Os homens representaram $62,7 \%$ do total da população estudada. A idade média dos funcionários foi de 35,1 anos, ( $D P=10,3)$. Quanto à escolaridade, somente $11,3 \%$ possuíam nível educacional menor que quatro anos e $42,2 \%$ entre cinco e oito anos. O ensino fundamental foi concluído por $14,3 \%$ dos entrevistados, o ensino médio por $25,1 \%$ e os de curso superior referiam-se, na sua maioria, às nutricionistas (dados não apresentados na Tabela). A renda familiar líquida foi de até dois salários-mínimos para 60\%; apesar disso, a expressiva maioria possuía casa própria, e 67,4\% tinham filhos (dados não apresentados na Tabela). Considerando os ex-fumantes, $81,2 \%$ não relataram uso corrente de tabaco.

A caracterização laboral demonstrou que $64,7 \%$ dos funcionários dos restaurantes populares encontraram-se concentrados nos cargos de Auxiliar de Serviço Geral e Copeira. Obteve-se para o tempo de trabalho em restaurantes indus- 
triais, uma média de 59,8 meses, tendo variado de um mínimo de dois meses e máximo de trinta anos. A maioria relatou permanecer na maior parte do tempo de trabalho na postura corporal de pé, $(90,4 \%)$, e responderam usufruir o horário de almoço (79,0\%) (dados não apresentados na Tabela). Somente $11,0 \%$ realizaram cursos de capacitação em instituições conveniadas com o comércio e indústria. Quanto ao treinamento realizado para ocupar o cargo, 60,5\% relataram ter recebido, e destes, $40 \%$ apontaram o próprio nutricionista do restaurante como responsável pelo treinamento (dados não apresentados na Tabela). A percepção de incômodos ambientais apre- sentou a seguinte ordem decrescente de importância: temperatura $(90,1 \%)$, ruído $(51,2 \%)$, esforço físico $(36,2 \%)$ e luminosidade $(10,5 \%)$. Somente $12,2 \%$ dos entrevistados relataram incômodo pessoal com a chefia (dado não apresentado na Tabela).

O estado geral de saúde foi considerado "muito bom" e "bom" em 83,8\% do total (Tabela 2). A prevalência de doenças relatadas que tinham diagnóstico médico foi de 15,0\% para DORT; 14,3\% para hipertensão arterial sistêmica; 12,7\% para gastrite; e, 2,1\% para diabete Mellitus tipo II. O acidente de trabalho corte foi relatado por $20,2 \%$ dos trabalhadores, seguido de contusão

Tabela 1. Características sócio-demográficas e laborais dos trabalhadores dos restaurantes populares do Estado do Rio de Janeiro. 2006/ 2007.

\begin{tabular}{|c|c|c|c|c|c|c|}
\hline Variáveis sócio-demograficas & $n=426$ & $\%$ & Variáveis laborais & & $n=426$ & $\%$ \\
\hline Sexo & & & Cargo & & & \\
\hline Masculino & 267 & 62,7 & $\mathrm{ASG}^{*}$ & & 154 & 36,1 \\
\hline Feminino & 159 & 37,3 & Copeira(o) & & 122 & 28,6 \\
\hline Faixa etária & & & Auxiliar de cozinha & & 39 & 9,1 \\
\hline Tána claria & 161 & 305 & Cozinheiro/Magarefe & & 36 & 8,5 \\
\hline Ate 29 anos & $\begin{array}{l}104 \\
180\end{array}$ & 38,5 & Estoquista & & 17 & 4,0 \\
\hline 30 a 44 anos & & 42,3 & Chefe de cozinha & & 6 & 1,4 \\
\hline 45 anos e mals & 82 & & Caixa & & 21 & 5,0 \\
\hline Cor & & & Auxiliar Administrativo & & 10 & 2,3 \\
\hline Negra & 110 & 25,8 & Nutricionista & & 21 & 5,0 \\
\hline Parda & 221 & 51,9 & Tempo de cozinha & & & \\
\hline Branca & 95 & 22,3 & Até 6 meses & & 48 & 11,3 \\
\hline Escolaridade & & & 7 a 12 meses & & 41 & 9,6 \\
\hline Menor que 4 anos & 48 & 11,3 & 13 a 24 meses & & 79 & 18,5 \\
\hline 5 a 8 anos & 180 & 42,2 & 25 meses e mais & & 258 & 60,6 \\
\hline 9 a 11 anos & 167 & 39,2 & Curso de capacitação & & & \\
\hline 12 anos e mais & 31 & 7,3 & Sim & & 47 & 11,1 \\
\hline Renda familiar $(R)$ & & & Não & & 379 & 88,9 \\
\hline R $\leq 1$ Salário-mínimo & 77 & 18,1 & Incômodos ambientais & & & \\
\hline 1 Salário-mínimo $R \geq 2$ Salários-mínimos & 178 & 41,8 & Calor & Sim & 384 & 901 \\
\hline R>2 Salários-mínimos & 171 & 40,1 & Calor & Não & 42 & 9,9 \\
\hline Situação conjugal & & & Ruído & Sim & 218 & 51,2 \\
\hline Solteiro & 136 & 32,4 & & Não & 208 & 48,8 \\
\hline Casado & 237 & 56,4 & Esforco físico & Sim & 154 & 36,2 \\
\hline Separado/viúvo & 53 & 11,2 & & Não & 272 & 63,8 \\
\hline Hábito de fumar & & & Luminosidade & Sim & 45 & 10,5 \\
\hline Nunca fumou & 268 & 62,9 & & Não & 381 & 89,5 \\
\hline Fuma atualmente & 80 & 18,8 & & & & \\
\hline Ex-fumante & 78 & 18,3 & & & & \\
\hline
\end{tabular}

ASG: auxiliar de serviços gerais. 
Tabela 2. Ausências por problemas de saúde, estado geral de saúde, relato de doenças, e acidentes de trabalho dos trabalhadores dos restaurantes populares do Estado do Rio de Janeiro. 2006/2007.

\begin{tabular}{|c|c|c|}
\hline Variáveis de situação de saúde & $n=426$ & $\%$ \\
\hline \multicolumn{3}{|c|}{ Ausências por problemas de saúde } \\
\hline Sim & 46 & 10,8 \\
\hline \multicolumn{3}{|l|}{ Estado geral de saúde } \\
\hline Muito bom & 155 & 36,4 \\
\hline Bom & 202 & 47,4 \\
\hline Regular e ruim & 69 & 16,2 \\
\hline \multicolumn{3}{|l|}{ Acidentes de trabalho } \\
\hline \multicolumn{3}{|l|}{ Corte } \\
\hline Sim & 86 & 20,2 \\
\hline Não & 340 & 79,8 \\
\hline \multicolumn{3}{|l|}{ Contusão } \\
\hline Sim & 68 & 16,0 \\
\hline Não & 358 & 84,0 \\
\hline \multicolumn{3}{|l|}{ Queimadura } \\
\hline Sim & 42 & 9,9 \\
\hline Não & 384 & 90,1 \\
\hline \multicolumn{3}{|l|}{ Choque elétrico } \\
\hline Sim & 30 & 7,0 \\
\hline Não & 396 & 93,0 \\
\hline \multicolumn{3}{|l|}{ Relato de doenças } \\
\hline DORT & 64 & 15,0 \\
\hline Hipertensão arterial & 61 & 14,3 \\
\hline Gastrite & 54 & 12,7 \\
\hline Diabetes mellitus tipo II & 9 & 2,1 \\
\hline
\end{tabular}

com $16,0 \%$. O relato de ausência no trabalho por motivo de doença, nas duas semanas anteriores à aplicação do questionário, foi de 10,8\%.

A Tabela 3 apresenta a análise estratificada por categorias ocupacionais, e mostra a distribuição dos diferentes estratos ocupacionais, segundo sexo, com uma proporção de homens nitidamente mais alta entre os de cargo de ASG. Na distribuição da idade, os cozinheiros apresentavam uma maior concentração $(74,9 \%)$ na faixa etária de maior de trinta anos. Maiores escolaridade e renda foram encontradas entre os administrativos. $O$ hábito de fumar foi mais expressivo entre os ASG. As únicas variáveis que não apresentaram diferenças estatisticamente significantes em suas distribuiç̧ões nos três grupos ocupacionais foram situação residencial e uso de bebida alcoólica.
Na Tabela 4 observa-se que o tempo de trabalho em cozinhas foi menor entre os ASG. Não usufruir do horário de almoço é uma condição descrita mais pelos administrativos. Realizar cursos na área de alimentação coletiva é uma situação pouco frequente entre os diferentes grupos ocupacionais, sendo menor nos ASG. O treinamento para realizar as atividades inerentes ao cargo também não é uma prática estabelecida para $50 \%$ dos trabalhadores. Aqueles trabalhadores que se encontraram, na maior parte do tempo do trabalho diário, ligados à execução do processo produtivo foram aqueles que mais se queixaram das condições ambientais de trabalho, principalmente no que se referiu ao calor e ao ruído. ASG apresentaram maior queixa da chefia, $18,8 \%$.

Na Tabela 5, a prevalência do estado geral de saúde referido não apresentou diferenças estatísticas entre os grupos ocupacionais. Entre os grupos que relataram apresentar doenças diagnosticadas por médicos, o grupo de cozinheiros foi o que expressou a maior proporção. A prevalência de acidentes de trabalho foi mais expressiva entre os ASG.

\section{DISCUSS Ã O}

A descrição do perfil dessa população permitiu identificar que os auxiliares de serviços gerais dos restaurantes industriais apresentam uma diferenciação social, laboral e de saúde, em relação aos grupos de administrativos e cozinheiros.

Nos restaurantes populares, os trabalhadores eram, na sua maioria, do sexo masculino, o que difere da maioria dos estudos. Em geral, os estudos realizados no setor de serviços de alimentação concentram-se em ambientes hospitalares. Isosaki ${ }^{13}$ encontrou em dois hospitais uma maior presença de trabalhadores do sexo feminino ( $n=123) 86 \%$ em um hospital público e $(n=162)$ $67 \%$ em um hospital privado. Casarotto \& Mendes $^{10}$ encontraram, em estudo realizado em qua- 
976 | O.B. AGUIAR et al.

Tabela 3. Distribuição sócio-demográfica dos trabalhadores dos restaurantes populares do Estado do Rio de Janeiro $2006 / 2007$.

\begin{tabular}{|c|c|c|c|c|c|}
\hline \multirow{2}{*}{ Variáveis sócio-demográficas } & \multirow{2}{*}{$\mathrm{n}$} & $\operatorname{ADM}(\mathrm{n}=69)$ & COP/COZ (n=203) & ASG $(n=154)$ & \multirow{2}{*}{$\begin{array}{c}\text { Total }(n=426) \\
p \text { valor }\end{array}$} \\
\hline & & \multicolumn{3}{|c|}{$\%$} & \\
\hline \multicolumn{6}{|l|}{ Sexo } \\
\hline Masculino & 267 & 46,4 & 48,7 & 88,3 & 0,00 \\
\hline Feminino & 159 & 53,6 & 51,2 & 11,7 & \\
\hline \multicolumn{6}{|l|}{ Idade } \\
\hline Até 29 anos & 164 & 44,9 & 25,1 & 53,2 & $0,00^{*}$ \\
\hline 30 a 44 anos & 180 & 46,4 & 47,3 & 33,7 & \\
\hline 45 anos e mais & 82 & 8,7 & 27,6 & 13,0 & \\
\hline \multicolumn{6}{|l|}{ Cor } \\
\hline Branca & 95 & 42.0 & 18,7 & 18,2 & $0,00^{*}$ \\
\hline Negra & 110 & 20,3 & 26,1 & 27,9 & \\
\hline Parda & 221 & 37.6 & 55,1 & 54,0 & \\
\hline \multicolumn{6}{|l|}{ Escolaridade } \\
\hline$\leq 4$ anos & 48 & 1,4 & 12,8 & 13,6 & 0,00 \\
\hline 5 a 8 anos & 180 & 15,9 & 45,8 & 49,3 & \\
\hline 9 anos e mais & 198 & 82,6 & 41,3 & 37,0 & \\
\hline \multicolumn{6}{|l|}{ Renda familiar $(R)$} \\
\hline R $\leq 1$ Salário-mínimo & 77 & 4,3 & 17,2 & 25,3 & $0,00^{*}$ \\
\hline 1 Salário-mínimo $<R \leq 2$ Salários-mínimos & 178 & 23,2 & 44,8 & 46,1 & \\
\hline R>2 Salários-mínimos & 171 & 72,4 & 37,9 & 28,6 & \\
\hline \multicolumn{6}{|l|}{ Situação residencial } \\
\hline Própria & 331 & 81,1 & 11,8 & 75,9 & 0,69 \\
\hline Alugada/outro & 95 & 18,8 & 22,2 & 24,0 & \\
\hline \multicolumn{6}{|l|}{ Situação conjugal } \\
\hline Solteiro & 136 & 36,2 & 27,1 & 36,3 & $0,00^{*}$ \\
\hline Casado & 237 & 56,5 & 53,7 & 57,8 & \\
\hline Separado/Viúvo & 53 & 7,2 & 19,2 & 5,8 & \\
\hline \multicolumn{6}{|l|}{ Hábito de fumar } \\
\hline Nunca fumou & 268 & 78,2 & 61,6 & 57,8 & $0,02^{*}$ \\
\hline Fuma atualmente & 80 & 13,0 & 17,2 & 23,4 & \\
\hline Ex-fumante & 78 & 8,7 & 21,2 & 18,8 & \\
\hline \multicolumn{6}{|l|}{ Uso de bebida alcoólica } \\
\hline Sim & 225 & 52,1 & 53,7 & 51,9 & 0,94 \\
\hline Não & 201 & 47,8 & 46,3 & 48,0 & \\
\hline
\end{tabular}

$p=0,05 ;{ }^{*}$ Teste de Fischer.

ADM: administrativo; COP/COZ: copeiro/cozinheiro; ASG: auxiliar de serviços gerais

tro restaurantes universitários e um hospital pediátrico $(n=256), 74 \%$ de mulheres. Na área hospitalar, este fato pode ser entendido pela necessidade da distribuição de refeições ocorrerem nas enfermarias e quartos, e, talvez, a presença de mulheres circulando pelas áreas das enfermarias possa diminuir o constrangimento, ou, que servir seja uma tarefa tipicamente feminina. No estudo realizado por Cavalli \& Salay ${ }^{11}$, em restaurantes comerciais nos municípios de
Campinas e Porto Alegre, 51,5\% e 48,5\% eram homens e mulheres, respectivamente. Estas proporções não apresentavam diferenças significativas entre si, entretanto uma maior presença masculina pode ser observada neste setor.

No estudo dos restaurantes populares $42,2 \%$ dos entrevistados encontrava-se com a escolaridade de ensino fundamental completo e incompleto e 39,2 \% com ensino médio completo e incompleto, e somente $11,1 \%$ possuíam curso 
Tabela 4. Distribuição laboral dos trabalhadores dos restaurantes populares do estado do Rio de Janeiro 2006/2007.

\begin{tabular}{|c|c|c|c|c|c|}
\hline \multirow{2}{*}{ Variáveis } & \multirow{2}{*}{$\mathrm{n}$} & $\operatorname{ADM}(n=69)$ & COP/COZ $(n=203)$ & ASG $(n=154)$ & \multirow{2}{*}{$\begin{array}{c}\text { Total }(\mathrm{n}=426) \\
p \text { valor }\end{array}$} \\
\hline & & \multicolumn{3}{|c|}{$\%$} & \\
\hline até 24 meses & 168 & 24,6 & 28,5 & 60,4 & 0,00 \\
\hline 25 meses e mais & 258 & 75,3 & 71,4 & 39,6 & \\
\hline Sim & 336 & 47,8 & 81,7 & 88,9 & 0,00 \\
\hline Não & 90 & 52,2 & 18,2 & 11,0 & \\
\hline \multicolumn{6}{|c|}{ Postura corporal no desenvolvimento da tarefa } \\
\hline Em pé & 385 & 56,5 & 96,0 & 98,0 & 0,00 \\
\hline Não & 379 & 81,1 & 88,6 & 92,8 & \\
\hline \multicolumn{6}{|c|}{ Percepção sobre incômodos ambientais (somente calor) } \\
\hline Sim & 384 & 84,0 & 91,6 & 90,9 & 0,17 \\
\hline Não & 42 & 15,9 & 8,3 & 9,1 & \\
\hline \multicolumn{6}{|c|}{ Percepção sobre incômodos ambientais (s/calor)1 } \\
\hline Sim & 126 & 73,9 & 70,4 & 68,8 & 0,74 \\
\hline Não & 300 & 26,1 & 29,5 & 31,2 & \\
\hline \multicolumn{6}{|c|}{ Percepção sobre incômodos com a chefia } \\
\hline
\end{tabular}

$p=0,05{ }^{*}$ Teste de Fischer; ${ }^{1}$ ruído, esforço, espaço físico.

ADM: administrativo; COP/COZ: copeiro/cozinheiro; ASG: auxiliar de serviços gerais.

Tabela 5. Distribuição das ausências por problemas de saúde, estado de saúde e acidentes de trabalho dos trabalhadores dos restaurantes populares do Estado do Rio de Janeiro. 2006/2007.

\begin{tabular}{|c|c|c|c|c|c|}
\hline Variáveis & $n$ & \multicolumn{3}{|c|}{$\%$} & $\begin{array}{c}\text { Total }(n=426) \\
p \text { valor }\end{array}$ \\
\hline Sim & 46 & 8,7 & 7,8 & 15,5 & 0,05 \\
\hline Não & 380 & 91,3 & 92,1 & 84,4 & \\
\hline Muito bom & 155 & 36,2 & 37,4 & 35,0 & 0,98 \\
\hline Bom & 202 & 46,3 & 46,8 & 48,7 & \\
\hline Regular e ruim & 69 & 17,4 & 15,7 & 16,2 & \\
\hline \multicolumn{6}{|c|}{ Acidente de trabalho } \\
\hline Presente & 149 & 37,6 & 43,3 & 22,7 & 0,00 \\
\hline Ausente & 277 & 62,3 & 56,6 & 77,3 & \\
\hline
\end{tabular}

$p=0,05$.

ADM: administrativo; COP/COZ: copeiro/cozinheiro; ASG: auxiliar de serviços gerais. 
de capacitação para produção de refeições. Cavalli \& Salay ${ }^{11}$ encontraram nível de escolaridades, em restaurantes comerciais, de 33,2\% para o nível médio completo e incompleto e $11 \%$ com relato de curso profissionalizante. A escassez de estudos na literatura sobre os trabalhadores do setor de alimentação coletiva ${ }^{10}$ dificulta a comparação com outros estudos, particularmente em relação à escolaridade, que sempre é apontada como uma dificuldade para estabelecimento da qualidade do setor $^{9,10}$. Apesar de somente $1 / 4$ dos trabalhadores dos restaurantes populares ter concluído o ensino médio, é importante ampliar a visão geral dos administradores de alimentação coletiva para a análise da questão da qualificação, pois quanto maior a escolarização, maiores serão as expectativas dos trabalhadores em relação à sua carreira. Gastos com formação e treinamento potencializam a profissionalização e comprometimento do trabalhador com a empresa.

A habilitação profissional do cozinheiro pode representar um trabalhador que desenvolveu habilidades para o preparo dos alimentos, e significaria uma maior renda, o que é um bom indicador de status profissional. Entretanto, constatou-se que somente $50 \%$ dos cozinheiros relataram alcançar renda familiar superior a dois salários-mínimos $(\mathrm{R} \$ 700,00)$, indicando um baixo prestígio social desta profissão. O piso salarial de cozinheiros iniciantes Campinas e Porto Alegre, no ano de 2001, estava em torno de $R \$ 405,00$, uma média de dois salários-mínimos no ano do estudo?.

Com referência ao uso de tabaco, os participantes apresentaram uma prevalência para o hábito de fumar de $23,4 \%$ entre os ASG. O uso de bebida alcoólica entre os trabalhadores de produção de refeições chama a atenção devido à sua alta prevalência nos três grupos. Em média, $50 \%$ afirmaram ter bebido, e 48,5\% afirmaram ter consumido cerveja ou outro tipo de bebida alcoólica nas duas últimas semanas.

Na região de Porto Alegre, Moreira et al. ${ }^{20}$ encontraram prevalência de tabagismo de $34,9 \%$. Griep et al. ${ }^{21}$ obtiveram estimativas menores em uma empresa bancária no Rio de Janeiro 29,5\% de fumantes. Castro et al. ${ }^{22}$ constataram, em investigação no restaurante universitário em Fortaleza, sobre adoção de estilo de vida compatível com o nível pressórico ótimo, a respeito do etilismo e tabagismo, $21(67,0 \%)$ e $25(78,0 \%)$ trabaIhadores, respectivamente, procuravam muitas vezes evitá-los.

Em 15 capitais brasileiras, a prevalência de tabagismo variou de $12,9 \%$ a $25,2 \%$ nas cidades estudadas no inquérito domiciliar sobre comportamentos de risco e morbidade referida de doenças e agravos não transmissíveis. No Brasil, estima-se que cerca de 200 mil mortes/ano são decorrentes do tabagismo ${ }^{23}$.

O tempo médio dos entrevistados no trabalho em refeições coletivas foi de cinco anos; entre os ASG - $60,4 \%$ deles possuem menos de 24 meses de atividades em restaurantes, demonstrando uma relação inversa com os cozinheiros e administrativos, em que $70 \%$ possuem mais de 25 meses. Casarotto \& Mendes ${ }^{13}$ encontraram um tempo médio de 8,18 anos, e Cavalli \& Salay ${ }^{11}$ encontraram entre os com maior tempo de serviço cinco anos e entre aqueles com menor tempo de serviço um ano, e estes afirmam que o setor absorve um contingente expressivo de pessoal desqualificado. A suposição de que, para realizar as atividades de higienização ambiental pelos ASG, não se necessita de habilidades pré-definidas ou conhecimentos técnicos, salienta a pouca atenção que é dada a este grupo funcional, sendo visto como mão-de-obra facilmente disponível no mercado.

Nos restaurantes populares, as ausências do trabalho nas duas semanas anteriores à pesquisa, por problemas de saúde, foram de 15,5\% entre os ASG, 7,8\% entre os COP/COZ e 8,7\% entre os $A D M$, sendo maior entre os homens do que em mulheres $12,7 \%$ e 7,5\%, embora não estatisticamente significante (RP:1,68; IC=95\%:de 0,90 a 3,13).

Na análise comparativa das proporções de dias perdidos dos hospitais públicos e privados, no trabalho de Isosaki ${ }^{15}$, foram significativos os 
efeitos de gênero, os dias perdidos entre homens foi $50 \%$ menor em relação às mulheres, nos dois hospitais; e tempo de trabalho apresentou-se $91 \%$ e $100 \%$ maior entre servidores com tempo menor que quatro anos e 5 a 14 anos, respectivamente, em relação a 15 anos e mais. Neste mesmo estudo, $61 \%$, dos registros nos Serviços de Medicina do Trabalho, foram relacionados a "doença própria" seguidos por $25 \%$ que foram por "motivos voluntários", e 14\% distribuídos entre acidente de trabalho, doença na família, legal e compulsório. Quando foram solicitadas sugestões para diminuição das faltas aos trabalhadores, tanto os absenteístas quanto os não absenteístas, sugeriram "melhorar as condições de trabalho".

O estudo populacional conduzido por Santana et al. ${ }^{24}$, sobre custos previdenciários e dias de trabalho perdidos ocasionados por acidentes de trabalho para o Estado da Bahia no ano de 2000, revelou que as maiores proporções de dias perdidos encontram-se nos ramos comércio/alojamento/alimentação, indústria da transformação e construção/eletricidade/gás. Os ramos com maior impacto na produtividade por acidentes de trabalho foram também os de comércio/alojamento/alimentação.

A rotatividade e o absenteísmo são as maiores queixas do setor e são apresentadas pelos pesquisadores como uma questão gerencial importante; entretanto, estes temas não têm sido alvo de exploração dos estudos ou associado às condições de trabalho e de saúde dos restaurantes industriais para se determinar a sua real extensão ${ }^{15}$.

Na presente pesquisa, entre os incômodos ambientais com o trabalho em unidades de alimentação coletiva, o calor foi relatado pelos COP/COZ e ASG. A luminosidade deficiente incomodava mais aos administrativos e o ruído afeta indistintamente todos os setores dos restaurantes, sendo mais percebido pelos ADM.

Matos \& Proença ${ }^{9}$ encontraram níveis de ruído acima do recomendado pela NR15-85dB(A) por oito horas nos setores de cocção e higieni- zação de bandejas. Os operadores dos setores de cocção e higienização de utensílios relatavam desconforto térmico. Abreu et al. ${ }^{14}$, também, encontraram na área de higienização de louças a maior exposição ao ruído e quanto ao calor, na área de cocção, excediam os limites recomendados.

Pela exigência do uso de equipamentos à base de calor ou frio, utilização abundante de água para o preparo dos alimentos, higiene da área física, o ambiente de produção de refeições pode nos levar erroneamente a considerar essas características inerentes ao ambiente de trabalho. Entretanto, normas técnicas de edificações e ergonomia são pouco consideradas e pouco desenvolvidas na construção destas áreas de trabalho, ocasionando todos esses desconfortos.

O principal prejuízo causado pelo ruído ocupacional é perda auditiva por indução de barulho, considerada uma desordem irreversível ${ }^{25}$. O ruído, também, pode ocasionar repercussões extra-audição como problemas cardiovasculares, endócrinos, gastrointestinais e mudanças de comportamento, tais como: fadiga mental e frustração? .

Nos restaurantes populares $20,2 \%$ dos entrevistados referiram ter se acidentado por corte, estes relatos estavam correlacionados ao fato de terem buscado assistência médica e, em algumas situações, ter tido licença médica. Seguido ao corte, contusão aparece com 16\% das informações obtidas, na maioria dos casos estes acidentes foram provocados pelo chão escorregadio ou por transportar inadequadamente material.

No estudo de acidentes de trabalho Isosaki ${ }^{15}$ encontrou uma proporção de $54 \%$ e $63 \%$ de acidentes ocorridos no próprio trabalho e nas primeiras horas de trabalho, no hospital público e privado, respectivamente. Os acidentes foram atribuídos às instalações no hospital público, provocando $58 \%$ das contusões, e aos equipamentos no hospital privado, provocando ferimentos com corte em $54 \%$ dos trabalhadores. Casarotto \& Mendes ${ }^{13}$ encontraram, em seu estu- 
do realizado em quatro restaurantes universitários e no serviço de nutrição de um hospital, uma proporção de 18,1\% de acidentes com cortes, 7,5\% de traumas, e quatro amputações de dedos relacionados à falta de utilização do êmbolo para empurrar a carne. Os acidentes de trabalho ocorridos nas unidades de alimentação e nutrição são, na maioria das vezes, atribuídos à natureza do trabalho, nos relatos de corte, contusão e queimaduras, pois estes somente teriam relevância se houvesse busca de assistência médica e afastamento do trabalho devido ao mesmo.

Os resultados dos estudos em unidades de alimentação apontam para a falta de planejamento nos projetos dos restaurantes industriais, a manutenção deficiente dos equipamentos e a organização do trabalho como fatores preponderantes para os acidentes de trabalho e doenças ocupacionais ${ }^{9,13}$. Área física inadequada, com cruzamentos de fluxos e espaços reduzidos em relação ao número de refeições produzidas; pisos escorregadios; exposição a altas temperaturas e ruído; manuseio de equipamentos elétricos, a vapor e a gás e de utensílios cortantes sem manutenção preventiva; ritmos intensos de trabalho e alta produtividade em tempo limitado, carregamento de pesos, em condições muitas vezes inadequadas de transporte e empilhamento são as situações recorrentes dos restaurantes industriais.

Nos restaurantes populares estudados, a prevalência de DORT, com relato de diagnóstico médico, foi de $15 \%$. Após um crescente aumento dos problemas de saúde relacionados ao trabalho, as lesões osteomusculares são os mais frequentes em todos os países, independente do seu patamar de industrialização ${ }^{26}$. As informações sobre as doenças pré-existentes foram coletadas de forma indireta, através da entrevista e não confirmada por exames clínicos. Esta informação pode apresentar uma limitação no estudo, por permitir uma subestimação nas prevalências de doenças consideradas silenciosas como a hipertensão e o diabetes. Entretanto, percepção da morbidade permite construir indicadores para o estado de saúde da população nos seus vários domínios. A percepção individual da saúde mostra-se importante por si, independente da presença objetiva de alguma doença ou incapacidade ${ }^{27}$.

No estudo de Casarotto \& Mendes ${ }^{13}$, $14,6 \%$ dos trabalhadores de restaurantes universitários e hospital apresentavam DORT. Geralmente, as atividades realizadas pela maioria dos trabalhadores são de pé, realizando movimentos contínuos e repetitivos, o que propicia que mantenham postura inadequada, principalmente se associada ao uso inadequado de equipamentos. O tempo de pausa durante a jornada de trabalho se restringe ao período do almoço; pausas curtas ou pausas preventivas, baseadas em estudos ergonômicos, não fazem parte da rotina desses funcionários ${ }^{9}$.

No estudo sobre condições de trabalho de alimentação coletiva, os autores ${ }^{9}$ verificaram que o cumprimento dos horários de entrada e saída eram cumpridos rigorosamente, enquanto os horários das refeições variavam de acordo com o ritmo das atividades diárias, constatando até cinco horas entre os intervalos das refeições. Intervenções ergonômicas e pausas nos ciclos de atividades não são medidas adotadas no ambiente laboral das unidades de refeições coletivas.

Considerando que estas medidas possam estar sendo subestimadas pelo efeito do trabalhador saudável, pode-se afirmar que os distúrbios osteomusculares crescem entre a população de trabalhadores. O efeito do trabalhador saudável é um tipo de viés de seleção, que pode subestimar a ocorrência dos problemas de saúde, pois os trabalhadores em atividade seriam mais saudáveis e aptos para o trabalho, comparados aos que estão fora do mercado de trabalho, justamente devido a problema de saúde.

Incômodo com a chefia apresentou uma maior prevalência entre os ASG, $(p=0,05)$. A dificuldade no relacionamento com as chefias apontadas nesse estudo parece ser pela maior dificuldade em estabelecer, com os ASG, uma postura mais justa e imparcial. Como sugeriram aqueles que faltaram ao trabalho "os absenteístas 
deveriam ser ouvidos pelas chefias" (expressão dos participantes) ${ }^{15}$.

Nos inquéritos epidemiológicos, seccionais, exposição e doença são avaliadas simultaneamente entre indivíduos, o que implica em limitações do estudo; geralmente, não é possível determinar se o fator em estudo (exposição) precedeu ou resultou da doença, podendo haver causalidade reversa, por exemplo. Esse tipo de estudo descritivo, que configura o perfil populacional, de um grupo específico de trabalhadores de restaurantes populares do Rio de Janeiro, limita a extensão dos resultados encontrados para a população geral.

\section{O N C L U S Ã O}

Os resultados do presente artigo sugerem que, apesar dos trabalhadores estarem expostos as mesmas condições ambientais de trabalho, o grupo ocupacional de Auxiliares de Serviços Gerais apresenta situações mais desfavoráveis que os demais grupos. Os ASG relataram maiores ausência do trabalho por problemas de saúde, prevalência de acidentes de trabalhos e desconforto térmico e de ruído. Esses fatores interferem no desenvolvimento das atividades da unidade de alimentação e podem gerar maior insatisfação com o setor de alimentação coletiva.

As questões ambientais no setor de alimentação coletiva têm sido reiteradas por diferentes estudos. Buscar soluções que amenizem, principalmente, o desconforto térmico e o ruído torna-se imprescindível nos setores de produção de refeições, para que se possa oferecer condições de trabalho à população de trabalhadores do setor. Enfatiza-se que as soluções não devem ficar restritas ou particularizadas para alguns setores, mas na estrutura ambiental como um todo. Investimentos em programas de redução de riscos ambientais focados no controle das fontes de emissão de ruído e desconforto térmico objetivam não somente manter os indivíduos em situação saudável, mas diminuir os acidentes de trabalho e o absenteísmo.
Estudos avaliando fatores explicativos das diferenças entre os cargos, encorajando metodologias alternativas, permitindo superar os limites deste estudo, podem precisar melhor essas diferenças.

\section{A GRADECIMENTOS}

Aos trabalhadores participantes, à Subsecretária e ao Superintendente da Secretaria de Estado da Família e da Assistência Social do Rio de Janeiro, responsáveis pela gestão dos restaurantes populares no ano de 2006, que autorizaram a realização deste trabalho.

\section{COLABORADORES}

O.B. AGUIAR contribuiu com a concepção do trabalho, definição da metodologia, orientação para coleta de dados, processamento e análise dos dados e elaboração do artigo. J.G. VALENTE e M.J.M. FONSECA contribuíram com a análise dos dados, elaboração e revisão do artigo.

\section{REFERÊ NCIAS}

1. Kon A. Mudanças recentes no perfil da distribuição ocupacional da população brasileira. R Bras Est Pop. 2006; 23(2):247-69.

2. Lee $\mathrm{D}$, Wolphin $\mathrm{KI}$. Intersectorial labor mobility and the growth of the service sector. Econometrica. 2006; 74(1):1-46.

3. Barros RP, Mendonça R. A absorção de mão-de-obra no setor de serviços. Dados. [Internet] 1997 [acesso jan 2008]; 40(1) Disponível em: <htpp// www.scielo.br/scielo.php?script=sci_arteex\& pid=S0011_52581997000100002\&nn=isso $>$.

4. Harnack L, Jeffery RW, Boutell KW. Temporal trends in energy intakes in the United States: an ecologic perspective. Am J Clin Nutr. 2000; 71(6):1478-84.

5. Kant AK, Graubard BI. Secular trends in patterns of self-reported food consumption of adult Americans:NHANES 1971-1975 to NHANES 19992002. Am J Clin Nutr. 2006; 84(5):1215-23.

6. Saelens BE, Glanz K, Sallis JF, Frank LD. Nutrition environment measures study in restaurants. Am J Prev Med. 2007; 32(4):273-81. 
7. Instituto Brasileiro de Geografia e Estatística. Pesquisa de orçamentos familiares 2002-2003. Rio de Janeiro: IBGE; 2004.

8. Associação Brasileira de Empresas de Refeições Coletivas. Mercado real de refeições. 2009 [acesso em 2009 out 21]. Disponível em: <http://www. aberc.com.br/mercadoreal.aspDMenu=21>.

9. Matos $\mathrm{CH}$, Proença RPC. Condições de trabalho e estado nutricional de operadores do setor de alimentação coletiva: um estudo de caso. Rev Nutr. 2003; 16 (4):493-502. doi: 10.1590/S1415-52732 003000400012 .

10. Collares LGT, Freitas CM. Processo de trabalho e saúde de trabalhadores de uma unidade de alimentação e nutrição: entre a prescrição e o real do trabalho. Cad de Saúde Pública. 2007; 23(12): 3011-20.

11. Cavalli SB, Salay E. Gestão de pessoas em unidades produtoras de refeições comerciais e a segurança alimentar. Rev Nutr. 2007; 20(6):657-667. doi: 10.1590/S1415-52732007000600008.

12. Noveletto D, Proença RPC. O planejamento do cardápio pode interferir nas condições de trabalho em uma unidade de alimentação e nutrição. Nutr Pauta. 2004; 65:36-40.

13. Casarotto RA, Mendes LF. Queixas, doenças ocupacionais e acidentes de trabalho em trabalhadores de cozinhas industriais. Rev Bras de Saúde Ocupacional. 2003; 28(107/108):119-26.

14. Abreu ES, Spinelli MGN, Araújo RMV. Fatores de risco ambiental para trabalhadores de unidades de alimentação e nutrição. Nutr Pauta. 2002; 57:46-9.

15. Isosaki M. Absenteísmo entre trabalhadores de serviço de nutrição e dietética de dois hospitais em São Paulo. Rev Bras de Saúde Ocupacional. 2003; 28(107/108):107-18.

16. Assunção AA. De La déficience a La gestion collective Du travail: lês troubles músculosquelettiques dansia restauration collective [thesis]. Ècole Pratique dês Hautes Etudes, EPHE. Paris; 1998.

17. Bertoldi CML, Proença RPC. Doença venosa e sua relação com as condições de trabalho no setor de produção de refeições. Rev Nutr. 2008; 21(4):447-54 doi: 10.1590/S1415-52732008000400009.

18. Borges LO, Argolo JCT. Adaptação e validação de uma escala de bem-estar psicológico para uso em estudos ocupacionais. Aval Psicol. 2002; 1(1):17-27.

19. Stata Corp. Statistical software release 9.0. Statistics / Data Analysis, Special Edition. Texas: StataCorp LP; 2005.

20. Moreira LB, Fuchs FD, Moraes RS, Bredemeir M, Cardozo S. Prevalência de tabagismo e fatores associados em área metropolitana da região Sul do Brasil. Rev Saúde Pública. 1995; 29(1):46-51.

21. Griep RH, Chór D, Camacho LAB. Tabagismo entre trabalhadores de empresa bancária. Rev Saúde Pública. 1998: 32(6):533-40.

22. Castro ME, Rolim MO, Mauricio TF. Prevenção da hipertensão e sua relação com o estilo de vida de trabalhadores. Acta Paul Enfer. 2005; 18(2):187-9.

23. Brasil. Ministério da Saúde. Secretaria de Vigilância em Saúde. Inquérito domiciliar sobre comportamentos de risco e morbidade referida de doenças e agravos não transmissíveis. Rio de Janeiro: INCA; 2003.

24. Santana VS, Araújo-Filho JB, Albuquerque-Oliveira PR, Barbosa-Branco A. Acidentes de trabalho: custos previdenciários e dias de trabalho perdidos. Rev Saúde Pública. 2006; 40(6):1004-12

25. Dias A, Cordeiro R. Attributable fraction of work accidents related to occupational noise exposure in a Southeastern city of Brazil. Cad Saúde Pública. 2007; 23(7):1649-55.

26. Wünsch Filho V. Perfil epidemiológico dos trabalhadores. Rev Bras Med Trab. 2004; 2(2):10317.

27. Theme Filha MM, Szwarcwald CL, Souza Junior PRB. Medidas de morbidade referida e inter-relações com dimensões de saúde. Rev Saúde Pública. 2008; 42(1):73-81.

Recebido em: 18/2/2009

Versão final reapresentada em: 29/10/2009 Aprovado em: 12/5/2010 